\title{
Characteristics and Correction of the Fading of Imaging Plate
}

\author{
Tomohiro SUZUKI ${ }^{\dagger}$, Chizuo MORI, Katsuo YANAGIDA, \\ Akira URITANI, Hiroshi MIYAHARA, \\ Department of Nuclear Engineering, Nagoya University*
}

Makoto YOSHIDA and Fumiaki TAKAHASHI

Department of Health Physics, Tokai Establishment, Japan Atomic Energy Research Institute**

(Received January 10, 1997)

\begin{abstract}
When the latent images formed by exposure with incident particles in the phosphor of Imaging Plate (IP) are irradiated with laser beam, photo-stimulated luminescence (PSL) is emitted and read through a photomultiplier. PSL intensity fades with increasing the elapsed time between the exposure and the PSL reading. When the IP is read successively, the PSL intensities decrease with the number of reading times. The tendency of the decreasing becomes gradual with increasing the elapsed time after exposure. The multiple successive reading method gives the means of the correction of the fading.
\end{abstract}

KEYWORDS: imaging plate, radioactivity, fading, laser beam, temperature dependence, alpha particles, beta particles, multiple successive reading method, stopping power, density, latent images, time dependence, radiation doses, irradiation, photo-stimulated luminescence

\section{INTRODUCTION}

Imaging Plate (IP) developed by Fuji Photo Film Co. is a new type of two-dimensional position-sensitive detector using photo-stimulable phosphor ( $\mathrm{BaFBr}: \mathrm{Eu}^{2+}$ ) in place of X-ray films. Imaging Plate is now used in various fields, because of its high sensitivity, good position resolution, large dynamic range and storage effect of latent images formed by incident particles ${ }^{(1)-(7)}$. Imaging Plate stores the image in the form of F-centers and $\mathrm{Eu}^{3+}$ ions in the phosphor. The photo-stimulated luminescence (PSL) is lead by putting IP into the reading device and irradiating it with the He-Ne laser beam (red light), and the information of energy deposition can be read out.

We pay attention to these characteristics and measure very small amount of radioactivity distributions such as rocks, meat and vegetables ${ }^{(5)(6)}$. It is necessary to expose for a long time to accumulate the latent image for the measurement of the weak radiations emitted by such materials. However, the PSL intensity fades with increasing the elapsed time between the exposure and the PSL reading ${ }^{(8)(9)}$. It is required to correct the fading to quantify the intensity of the low level radiations. The

\footnotetext{
* Furo-cho, Chikusa-ku, Nagoya 464-01.

** Tokai-mura, Naka-gun, Ibaraki-ken 319-11.

† Corresponding author, Tel. +81-52-789-4695,

Fax. +81-52-789-5127,

E-mail: h952402d@eds.ecip.nagoya-u.ac.jp
}

purpose of this study is to measure the characteristics of the fading and to find the method of the correction for the fading.

\section{EXPERIMENTS}

Imaging Plate used in these experiments is BAS III produced by Fuji Photo Film Co. It has a protective mylar film with $10 \mu \mathrm{m}$ thickness over photo-stimulable phosphor (BaFBr: $\mathrm{Eu}^{2+}$ ) of $150 \mu \mathrm{m}$ thickness which is painted on a plastic backing of $250 \mu \mathrm{m}$ thickness. As radiation sources, ${ }^{241} \mathrm{Am}(\alpha$-particle emitter, $5.4 \mathrm{MeV})$ with a mylar film cover of $3 \mu \mathrm{m}$ thickness, ${ }^{90} \mathrm{Sr}-{ }^{90} \mathrm{Y}(\beta$ particle emitter, $2.27 \mathrm{MeV})$ and ${ }^{147} \mathrm{Pm}(\beta$-particle emitter, $226.4 \mathrm{keV}$ ) with a mylar film cover of $12 \mu \mathrm{m}$ thickness were used. Imaging Plate was kept at $23^{\circ} \mathrm{C}$ and $5{ }^{\circ} \mathrm{C}$ to find the effect of temperature on the fading. The exposed IP was read out with an IP reading system BAS2000 produced by Fuji Photo Film Co. Imaging Plate was successively read out by putting it into BAS2000 in the dark room to avoid the irradiation with visible light.

The latent image caused by background radiations is accumulated with the elapsed time between the exposure and the reading procedure. The background effect was removed by subtracting the PSL intensity of the region without exposure on the same IP every reading procedure. 


\section{Experimental Results}

\section{The First Reading Intensity and the} Elapsed Time after Exposure

Imaging Plate was exposed with the particles ( $\alpha$ - or $\beta$-particles) for $5 \mathrm{~min}$, left at $23^{\circ} \mathrm{C}$ and $5{ }^{\circ} \mathrm{C}$ for a certain time and read out once with BAS2000. Figure 1 shows the PSL intensity of the first reading as a function of the elapsed time $(t)$ between the exposure and the reading. The intensity faded rapidly in a few hours after exposure and gradually after that. For $\alpha$-particle incidences, PSL intensities faded to $61 \%$ and $71 \%$ in $5 \mathrm{~h}$ when IP was kept at $23{ }^{\circ} \mathrm{C}$ and $5{ }^{\circ} \mathrm{C}$, respectively. For ${ }^{90} \mathrm{Sr}^{90} \mathrm{Y}$ $\beta$-particle incidences, PSL intensities faded to $69 \%, 75 \%$ in the same condition to $\alpha$-particle incidences. On the other hand when IP was read 5 days after, PSL intensities faded to $33 \%, 42 \%$ for $\alpha$-particles and, $50 \%, 60 \%$ for $\beta$-particles, respectively. The fading depends on the kind of incident particles, which is larger for $\alpha$-particle incidences than for $\beta$-particles. It also depends on the temperature. As a matter of course, the fading is slow at lower temperature.

\section{The Decreasing of PSL Intensities with the Number of Reading Times}

A single irradiation with laser beam cannot completely read out a latent image and the residual image can be read out by successive irradiation with laser beam. The decreasing of PSL intensities with the number of reading times depends on the kind and energy of incident particles ${ }^{(5)}$ as shown in Fig. 2 in the case of 5 min exposure at $23^{\circ} \mathrm{C}$ and reading just after exposure. The PSL intensity ratios of the second reading to the first one $(\operatorname{PSL}(n=2, t=0) / \operatorname{PSL}(n=1, t=0))$ are $0.18,0.30$ and 0.38 in the cases of ${ }^{241} \mathrm{Am} \alpha$-particle, ${ }^{147} \mathrm{Pm} \beta$ particle and ${ }^{90} \mathrm{Sr}^{-90} \mathrm{Y} \beta$-particle incidences, respectively.

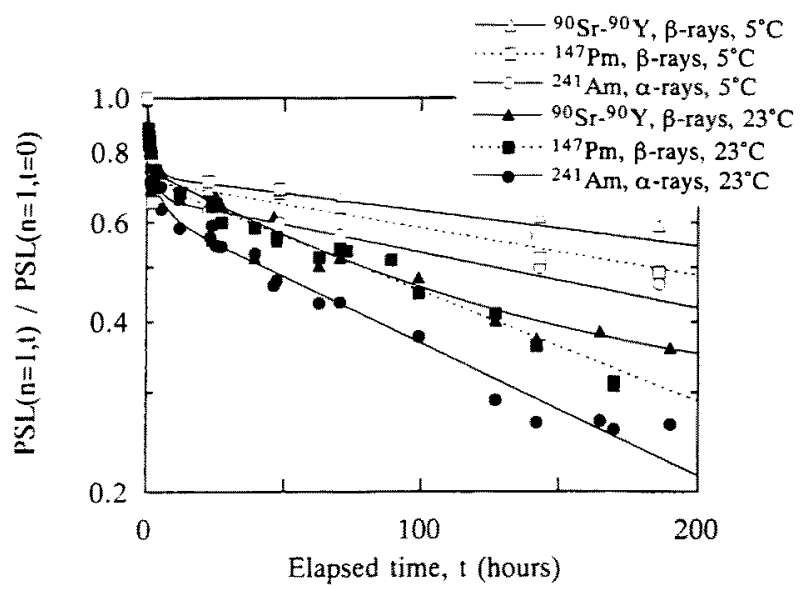

Fig. 1 The fading of the first reading intensity as a function of the elapsed time between exposure and reading

The ordinate is normalized at the time of zero (substantially about one minute).

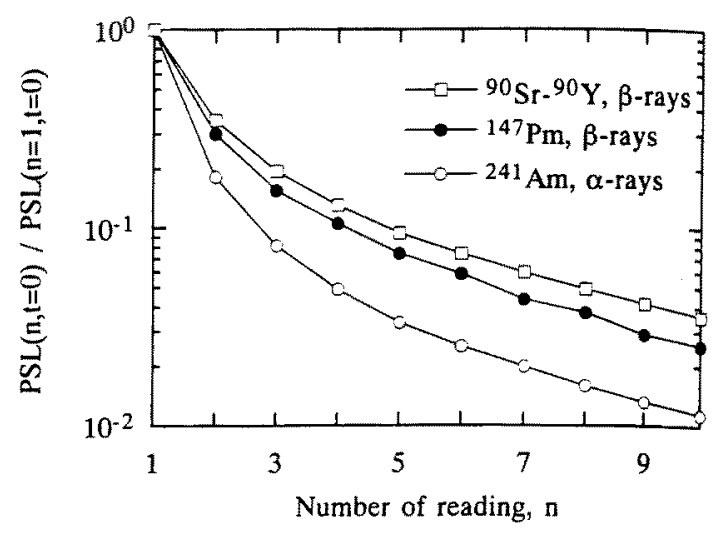

Fig. 2 The PSL intensity ratio as a function of the number of reading times with the multiple successive reading method

The ratio depends on the kind and energy of incident particles.

The ratio becomes smaller with increasing the stopping power (or with decreasing the range) of incident particles.

The tendency of the decreasing of PSL intensities with the number of reading times also depends on the elapsed time between the exposure and the reading. Figure 3 shows the tendency in the case that IP was kept at $23^{\circ} \mathrm{C}$, which becomes more gradual with increasing the elapsed time. Similar tendency was observed at $5^{\circ} \mathrm{C}$ though the change of the tendency of the decreasing is faster with increasing the temperature. For $\alpha$-particle incidences, the PSL intensity ratio of the second time reading to the first one $(\operatorname{PSL}(n=2, t) / \operatorname{PSL}(n=1, t))$ was 0.18 when IP was read just after exposure ( $t=0$, substantially about $1 \mathrm{~min}$ ), 0.24 at $t=1.3 \mathrm{~h}$ and 0.38 at $t=190 \mathrm{~h}$ as shown in Fig. 3(a). In the same way, the ratios were $0.30,0.36$ and 0.42 for ${ }^{147} \mathrm{Pm} \beta$-particle incidences and $0.38,0.42$ and 0.48 for ${ }^{90} \mathrm{Sr}^{90} \mathrm{Y} \beta$-particle incidences, respectively as shown in Figs. 3(b) and (c). The change of the successive reading curves with the elapsed time shown in Fig. 3 is also dependent on the kind of incident particles. The curves become gradual more quickly for $\alpha$-particle incidences than for $\beta$-particle incidences.

Figure 3 is revised to Fig. 4 so that the ordinate in Fig. 4 means the absolute PSL intensity per a single incident particle. For $\alpha$-particle incidences, the PSL intensity at the tenth time reading was $0.0035 \mathrm{PSL} /$ particle when IP was read just after exposure $(t=0)$, is was $0.0052 \mathrm{PSL} / \mathrm{particle}$ at $t=1.3 \mathrm{~h}$ and $0.0044 \mathrm{PSL} /$ particle at $t=190 \mathrm{~h}$. In some cases, it is clear for $\alpha$-particle incidences, PSL intensities at the latter part of reading times left for a long time after exposure are larger than those left for a short time, which is very interesting.

\section{Correction of the Fading}

Because it was observed that the tendency of the decreasing of PSL intensities with the number of reading times depended on the elapsed time after expo 

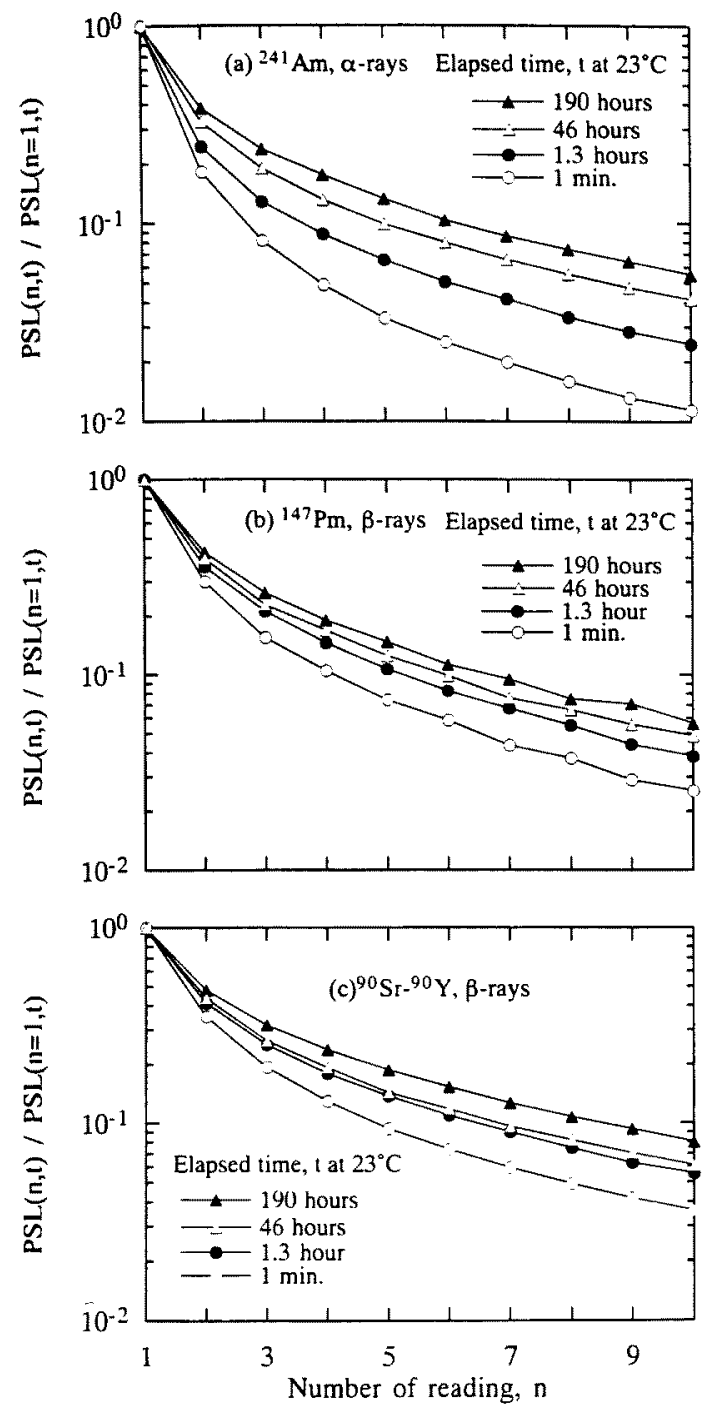

Fig. 3 The ratio of PSL intensity as a function of the number of reading times

IP was kept at $23^{\circ} \mathrm{C}$ in the elapsed time after exposure. The ordinate is normalized at the first reading intensity. (a) $\alpha$-particle incidences, (b) ${ }^{147} \mathrm{Pm} \beta$-particles, (c) ${ }^{90} \mathrm{Sr}-{ }^{90} \mathrm{Y}$ $\beta$-particles. The ratio also depends on the elapsed time after exposure.

sure, the faded intensity might be estimated by the multiple successive reading method. The results shown in Fig. 1 and Fig. 3 can be rewritten as shown in Fig. 5. The abscissa of Fig. 5 is the ratio of the PSL intensity of the first time reading to the intensity just after exposure $(\operatorname{PSL}(n=1, t) / \operatorname{PSL}(n=1, t=0))$, which is shown in the ordinate of Fig. 1. Substantially $t=0$ means about $1 \mathrm{~min}$. The ordinate of Fig. 5 is the PSL intensity ratio of the second or third reading to the first one $(\operatorname{PSL}(n=2, t) / \operatorname{PSL}(n=1, t)$ or $\operatorname{PSL}(n=3, t) / \mathrm{PSL}$ $(n=1, t))$, which is shown in the ordinate of Fig. 3. The relations between $\operatorname{PSL}(n=2, t) / \operatorname{PSL}(n=1, t)$ or PSL $(n=3, t) / \operatorname{PSL}(n=1, t)$ and $\operatorname{PSL}(n=1, t) / \operatorname{PSL}(n=1, t=0)$
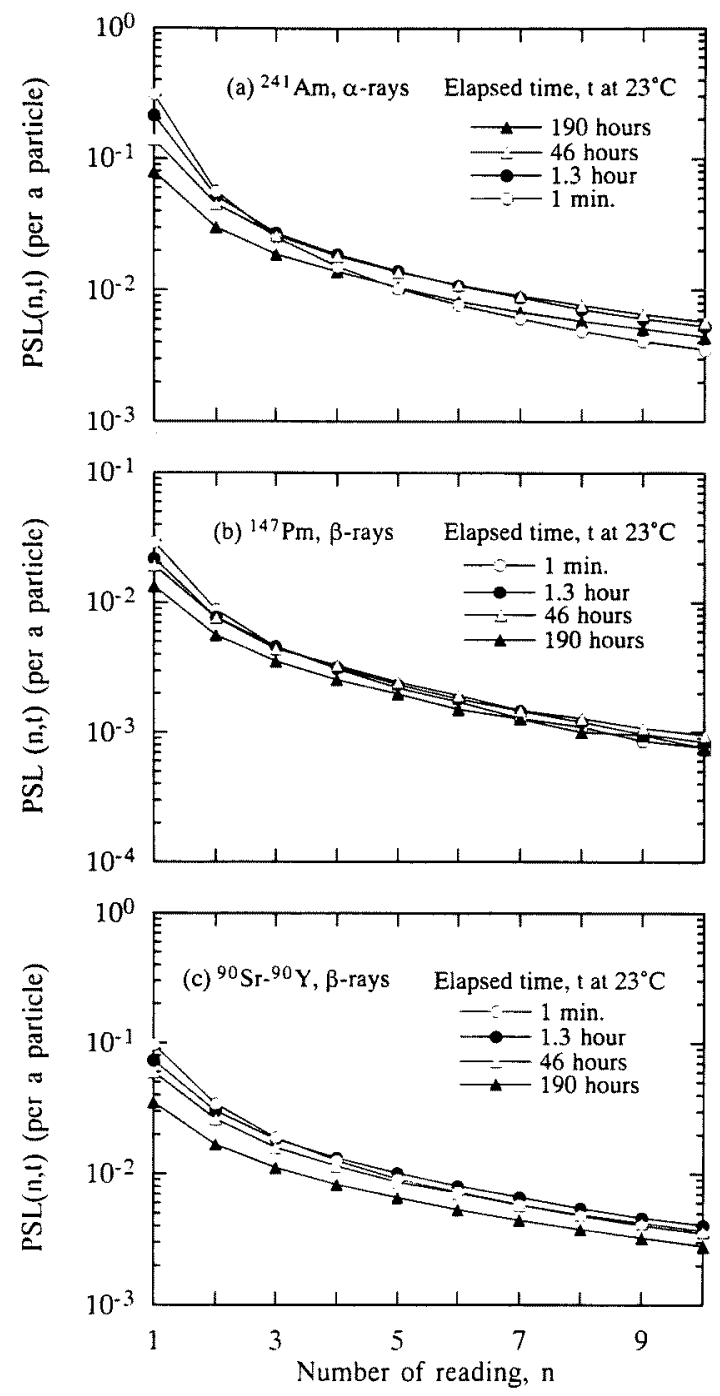

Fig. 4 The ordinates in Fig. 3 are rewritten to the absolute PSL intensity per a single incident particle.

(a) $\alpha$-particle incidences, (b) ${ }^{147} \mathrm{Pm} \quad \beta$ particles, (c) ${ }^{90} \mathrm{Sr}^{-}{ }^{90} \mathrm{Y} \beta$-particles

show straight lines. The dotted lines show the departures of $\operatorname{PSL}(n=1, t) / \operatorname{PSL}(n=1, t=0)$ by $\pm 10 \%$ from the solid line. The exposure conditions are different; IP was exposed once and kept at $23^{\circ} \mathrm{C}$ (in the figure), exposed three times and kept at $23^{\circ} \mathrm{C}(\boldsymbol{\Delta})$, exposed once and kept at $5^{\circ} \mathrm{C}(\bigcirc)$ lie along almost the same straight lines. The strong correlation is observed between the fading and the multiple successive reading curve. By comparing the ratio of successive readings of an IP with unknown exposure with the relations shown in Fig. 5 which has beforehand been measured, the fading of the first reading intensity can be estimated. If the temperature condition in the elapsed time between the exposure and the reading is equal to that of beforehand measurement such as shown in Figs. $1-5$, the elapsed time $t$ will be estimated by referring Fig. 1. But if the condition is 

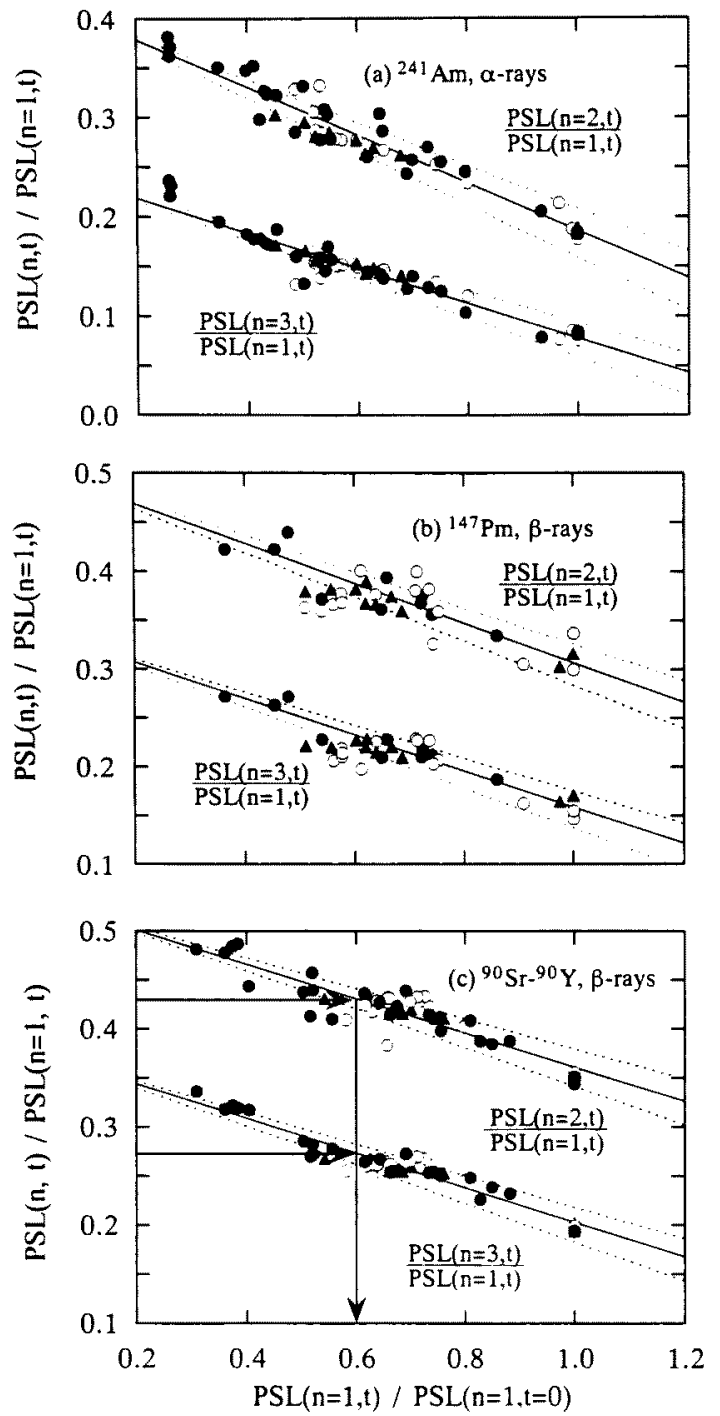

Fig. 5 Correction of the faded intensity by the multiple successive reading method

The abscissa is the first reading intensity normalized at the intensity just after exposure. The ordinate is the ratio $\left(L_{2} / L_{1}\right.$ or $\left.L_{3} / L_{1}\right)$ of PSL intensity of the second or third reading to the first reading intensity. (a) $\alpha$-particle incidences, (b) ${ }^{147} \mathrm{Pm} \beta$-particles, (c) ${ }^{90} \mathrm{Sr}-{ }^{90} \mathrm{Y} \beta$-particles; a single exposure and kept at $23^{\circ} \mathrm{C}(\mathbf{O})$, three times exposure and kept at $23^{\circ} \mathrm{C}(\mathbf{A})$, a single exposure and kept at $5^{\circ} \mathrm{C}(\mathrm{O})$

different, the elapsed time $t$ cannot be estimated with this method.

As an example, it is assumed that for ${ }^{90} \mathrm{Sr}^{90} \mathrm{Y} \beta$ particle incidences IP is read three times and the PSL intensity ratio $\operatorname{PSL}(n=2, t) / \operatorname{PSL}(n=1, t)$ is obtained to be 0.43 and $\operatorname{PSL}(n=3, t) / \operatorname{PSL}(n=1, t)$ is 0.28 . The straight lines in Fig. 5(c) are expressed by

$$
\frac{\operatorname{PSL}(n=1, t)}{\operatorname{PSL}(n=1, t=0)}=3.05-5.69 \times \frac{\operatorname{PSL}(n=2, t)}{\operatorname{PSL}(n=1, t)}
$$

$$
\frac{\operatorname{PSL}(n=1, t)}{\operatorname{PSL}(n=1, t=0)}=2.14-5.67 \times \frac{\operatorname{PSL}(n=3, t)}{\operatorname{PSL}(n=1, t)}
$$

The corresponding value of $\operatorname{PSL}(n=1, t) / \operatorname{PSL}(n=1, t=0)$ is found to be 0.60 by referring Fig. 5 (c), Eqs.(1) and (2), which means that the first reading intensity at $t=0 \operatorname{PSL}(n=1, t=0)$ should be $\operatorname{PSL}(n=1, t) / 0.60$ with the known value of $\operatorname{PSL}(n=1, t)$ observed at present.

As shown above, $\operatorname{PSL}(n=1, t) / \operatorname{PSL}(n=1, t=0)$ can be calculated from the measured $\operatorname{PSL}(n=2, t) / \operatorname{PSL}(n=1, t)$ and Eq. (1). We put that the calculated PSL $(n=1, t) / \mathrm{PSL}$ $(n=1, t=0)$ is $C$ and the measured $\operatorname{PSL}(n=1, t) / \mathrm{PSL}$ $(n=1, t=0)$ shown in the abscissa of Fig. 5 is $M$. The ratio $C / M$ must be unity. However, calculated $C / M$ values for all the measured data scattered around unity with the standard deviation of 0.089 . Therefore the fading can be corrected with an accuracy of about $10 \%$.

\section{Discussions}

We consider that the results obtained above will be caused by the decreasing of the density of latent image (F-centers and $\mathrm{Eu}^{3+}$ ions) for two reasons below. One is that the images made by incident particles disappear by the process of emission or no emission de-excitation. The density of latent images decreases through the process. The other is that the images along the particle tracks diffuse and the density decreases.

Figure 6 shows the typical energy level scheme of IP (BaFBr: $\left.\mathrm{Eu}^{2+}\right)^{(10)}$. An electron excited by a photon (He-Ne laser beam) from F-center to the conduction band has a probability whether it recombines with a trapped hole $\left(\mathrm{Eu}^{3+}\right.$ ion) and emits PSL (probability $p$ in the figure) or it is again trapped by an $\mathrm{F}^{+}$-center and becomes an F-center (probability $q$ in the figure). The probability $p$ to emit PSL depends on the densities of both trapped holes and $\mathrm{F}^{+}$-centers. If there are trapped holes with large density and $\mathrm{F}^{+}$-centers with small density, such as the case for just after exposure, the probability of emission will be large (that is to say, $p$ is large) and many of the electrons will be read out with a sin-

(a)

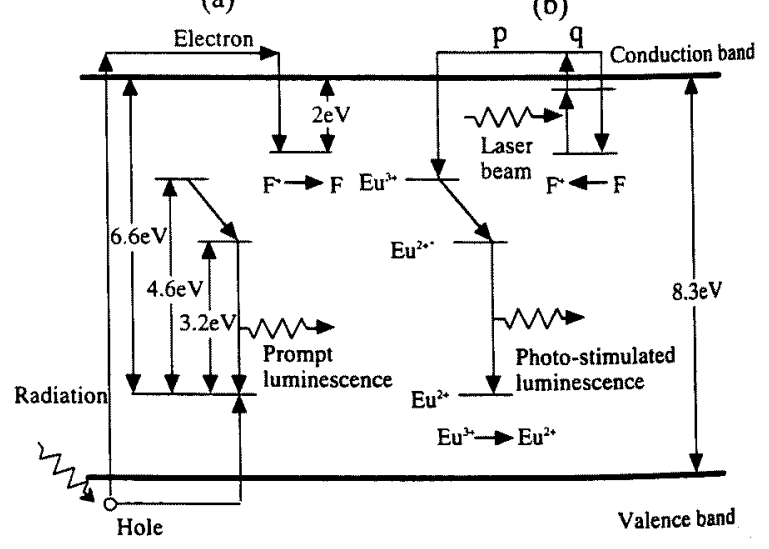

Fig. 6 Typical energy level scheme of $\mathrm{BaFBr}: \mathrm{Eu}^{2+}$

(a) Particle incidence, (b) Optical stimulation ${ }^{(10)}$ 
gle reading. Therefore, the PSL intensities of the latter successive readings are relatively small. If there are trapped holes with small density and $\mathrm{F}^{+}$-centers with large density such as the case with long elapsed time after exposure, the probability of emission will be small (that is to say, $q$ is large) and many of the electrons will be again trapped by F-centers, since we consider that trapped holes and $\mathrm{F}^{+}$-centers diffuse after exposure. Therefore, the PSL intensities of the latter readings are relatively larger. This explanation can be supported by the fact that the ratio of PSL intensity to prompt emission intensity is smaller for $\alpha$-particle incidences with large specific ionization $-d E / d x$ than for $\beta$-particle incidences as shown in Fig. $\mathbf{7}^{(11)}$.

The diffusion velocity of the densely distributed images is larger than that of the thin images because the velocity is proportional to the gradient of the density. Therefore, the diffusion velocity is larger for $\alpha$-particle incidences, and the decreasing of PSL intensity both with the elapsed time shown in Fig. 1 and with the number of successive reading times shown in Fig. 3 are larger for $\alpha$-particle incidences than for $\beta$-particle incidences. The mechanism of diffusion of electrons and holes may be thermal excitation from shallow trappings like a kind of small lattice distortion or tunneling effect in the time between the exposure and the reading. The details of the mechanism will be obtained by the further investigations.

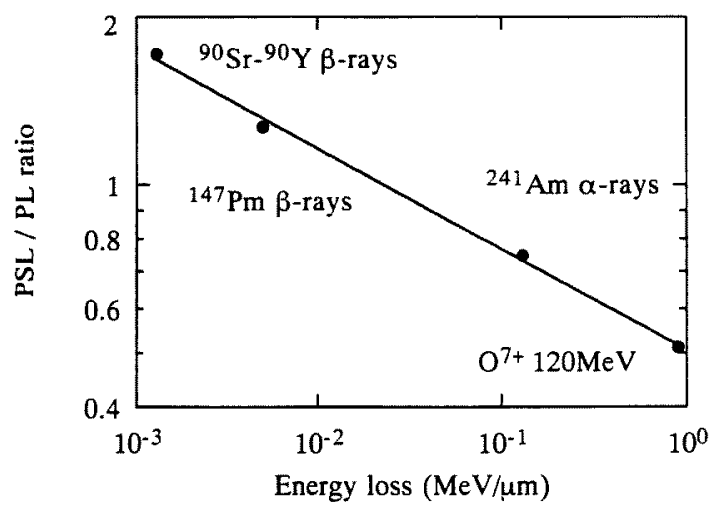

Fig. 7 The ratio of PSL intensity to prompt luminescence (PL) intensity as a function of the specific energy loss of incident particles ${ }^{(11)}$

\section{Conclusions}

Photo-stimulated luminescence intensity of the first reading fades with increasing the elapsed time between the exposure and the reading. The tendency of the fading depends on the temperature and the kind of incident particles. The decrease of PSL intensities with the number of successive reading times depends on the kind of incident particles and on the elapsed time after exposure.

We consider that such phenomena would be caused by the density of the latent image (F-centers and $\mathrm{Eu}^{3+}$ ions). The images diffuse or disappear with the elapsed time and the density decreases. Hence, the ratio of the first reading intensity to all image intensity decreases and PSL intensities of the latter part readings relatively increase. These phenomena are notable for $\alpha$-particle incidences because of the large stopping power. The difference of the decrease of PSL intensities with the number of successive reading times makes it possible to correct the fading of PSL intensity.

\section{ACKNOWLEDGMents}

The authors would like to express their thanks to Mr. K. Takahashi and his coworkers, Fuji Photo Film Co., Ltd., for their valuable discussions.

$$
\text { -References- }
$$

(1) Amemiya, Y, et al.: Nucl. Instrum. Methods, A266, 645 (1988).

(2) Ito, M., Amemiya, Y.: Nucl. Instrum. Methods, A310, 369 (1991).

(3) Katto, M., et al.: Rev. Sci. Instrum., 64, 319 (1993).

(4) Niimura, N., et al.: Nucl. Instrum. Methods, A349, 521 (1994).

(5) Mori, C., et al.: Nucl. Instrum. Methods, A353, 371 (1994).

(6) Mori, C, et al.: J. Radioanal. Nucl. Chem., 206, 263 (1996).

(7) Takahashi, K., et al.: J. Lumin., 31\&32, 266 (1984).

(8) Oikawa, T., et al.: J. Electron Microsc., 43, 402 (1994).

(9) Mori, C., et al.: Nucl. Instrum. Methods, A339, 278 (1994).

(10) Iwabuchi, Y., et al.: Jpn. J. Appl. Phys., 33, 178 (1994).

(11) Suzuki, T., et al.: To be published in Nucl. Instrum. Methods. 